\title{
ERRATUM
}

Nathaniel E. Ostrom · Hunter J. Carrick

Michael R. Twiss · Leah Piwinski

\section{Evaluation of primary production in Lake Erie by multiple proxies}

Published online: 16 August 2005

(C) Springer-Verlag 2005

\section{Oecologia (2005) 144:115-124}

Due to an unfortunate error entries were incorrectly underlined in the final column of Table 3. The correct table is printed below.

Table 3 Results from a blocked, one-way analysis of variance (ANOVA) comparing primary production estimated derived from three independent analytical methods in each of four lake regions

\begin{tabular}{|c|c|c|c|c|c|}
\hline \multicolumn{3}{|l|}{ Blocked Factor } & \multirow{2}{*}{\multicolumn{3}{|c|}{$\begin{array}{l}\text { Tukey's pair-wise } \\
\text { comparison } \\
\text { (least to greatest) }\end{array}$}} \\
\hline \multirow{2}{*}{$\frac{\text { Lake region, station }}{\text { Western, } 1163}$} & \multirow{2}{*}{$\frac{\text { Depth }(\mathrm{m})}{3}$} & \multirow{2}{*}{$\frac{F \text { statistic }}{41.3^{* *}}$} & & & \\
\hline & & & ${ }^{18} \mathrm{O}-\mathrm{PP}$ & LD-PP & ${ }^{14} \mathrm{C}-\mathrm{PP}$ \\
\hline \multirow{3}{*}{$\begin{array}{l}\text { Central (west), } \\
43 \mathrm{M}\end{array}$} & 5 & $953.2^{* *}$ & ${ }^{18} \mathrm{O}-\mathrm{PP}$ & ${ }^{14} \mathrm{C}-\mathrm{PP}$ & LD-PP \\
\hline & 16 & 2.0 & ${ }^{18} \mathrm{O}-\mathrm{PP}$ & LD-PP & ${ }^{14} \mathrm{C}-\mathrm{PP}$ \\
\hline & 20 & $12.5^{*}$ & ${ }^{18} \mathrm{O}-\mathrm{PP}$ & ${ }^{14} \mathrm{C}-\mathrm{PP}$ & $\underline{\text { LD-PP }}$ \\
\hline \multirow{4}{*}{$\begin{array}{l}\text { Central (east) } \\
78 \mathrm{M}\end{array}$} & 5 & $65.0^{* *}$ & ${ }^{18} \mathrm{O}-\mathrm{PP}$ & ${ }^{14} \mathrm{C}-\mathrm{PP} \mathrm{I}$ & LD-PP \\
\hline & 10 & 0.5 & ${ }^{18} \mathrm{O}-\mathrm{PP}$ & ${ }^{14} \mathrm{C}-\mathrm{PP}$ & LD-PP \\
\hline & 15 & $79.6^{* *}$ & ${ }^{18} \mathrm{O}-\mathrm{PP}$ & ${ }^{14} \mathrm{C}-\mathrm{PP}$ & $\underline{\text { LD-PP }}$ \\
\hline & 20 & 1.4 & ${ }^{18} \mathrm{O}-\mathrm{PP}$ & ${ }^{14} \mathrm{C}-\mathrm{PP}$ & LD-PP \\
\hline $\begin{array}{l}\text { Eastern, } \\
15 \mathrm{M}\end{array}$ & 10 & $88.5^{* * *}$ & ${ }^{18} \mathrm{O}-\mathrm{PP}$ & $\underline{\text { LD-PP }}$ & ${ }^{14} \mathrm{C}-\mathrm{PP}$ \\
\hline
\end{tabular}

Treatments joined by underlining were not significantly different from one another when assessed using a Tukey's pair-wise comparison $(P<0.05)$. Where $* P<0.01, * * P<0.001$

The online version of the original article can be found at http:// dx.doi.org/10.1007/s00442-005-0032-5

N. E. Ostrom $(\bowtie) \cdot$ L. Piwinski

Department of Geological Sciences, Michigan State University, East Lansing, MI 48824, USA

N. E. Ostrom

Center for Global Change and Earth Observations,

Michigan State University, East Lansing, MI 48824, USA
H. J. Carrick

School of Forest Resources, Pennsylvania State University, 8B Ferguson Building, University Park, PA 16802, USA

M. R. Twiss

Department of Biology, Clarkson University, Potsdam, NY 13699-5805, USA 